\title{
Lattice transformations and subunit conformational changes in phage capsid maturation
}

\author{
DAVID C. GOSSARD $\uparrow \uparrow$ and JONATHAN KING $\neq *$ \\ $\dagger$ Department of Mechanical Engineering, Massachusetts Institute of Technology, Cambridge, MA 02139, USA \\ $\ddagger$ Department of Biology, Massachusetts Institute of Technology, Cambridge, MA 02139, USA

\begin{abstract}
A general feature of the pathways for the assembly of double-stranded DNA phages and viruses is the assembly of coat and scaffolding subunits into a precursor shell or procapsid, followed by packaging of the genomic DNA into the shell. Coupled to this DNA packaging process is the loss of the scaffolding subunits and expansion and re-organization of the procapsid lattice to the lattice of the mature virus. Such lattice transitions have also been observed with adenoviruses and herpesviruses. In re-organizing into the mature capsid lattice, each subunit of the precursor lattice must change its conformation, or its relationship with its neighbours, or both. We briefly review here recent structural data for phages P22 and HK97, and describe the motions and conformational changes associated with this lattice transition. Possible functions of such constrained transformations within the virus life-cycle are discussed.
\end{abstract}

Keywords: Capsid; Procapsid; Lattice; Virus; Subunits

Msc: Geometry

\section{Introduction}

Viruses encapsulate their genomic material in a protective shell comprising a large number of identical coat proteins. The recognition that these subunits were generally organized into a variant of icosahedral symmetry was an important pillar of structural virology [2]. A subsequent surprising discovery, emerging from investigations of the assembly of bacterial viruses, was that a precursor protein shell is assembled empty of DNA and subsequently, the genomic DNA is pumped into the precursor shell $[8,11]$. This is true not only for phages with double-stranded DNA, but also for animal viruses including adenoviruses and herpesviruses.

The precursor procapsids of double stranded DNA phages have been studied in considerable detail. In general, they contain the capsid subunits, together with a large number of scaffolding subunits, which are absent from the mature capsid. These are needed for the assembly of the procapsid shell, but are absent from the mature virion [8,11]. In addition, the precursor shells contain a unique portal complex, situated at one of the 12 vertices, which is the channel through which the DNA is packaged and subsequently exits from the mature shell. The organization of the scaffolding shell within the procapsid has not been revealed by cryo-EM studies, implying that the molecules are not organized with icosahedral symmetry.

In the course of the loss of scaffolding subunits, and entrance of DNA, the precursor shells expand from a spherical geometry to the mature icosahedral geometry as shown in figure 1 . These processes were first studied with phages T4 and lambda using optical diffraction of negatively stained micrographs [6,13]. Subsequently, these processes have been studied at higher resolution by cryo-electron microscopy $[1,7,9,10,12]$. For phage P22, the hexagonal array of subunits at local six-fold axes are distinctively skewed, with a hole in the centre. The hole is presumably the site of exit of scaffolding molecules prior to DNA packaging. As the lattice transitions to the capsid, the holes in the centre of the hexamers close and the hexamers become less skewed and more regular as shown in figure 2.

\section{Maturation transition: procapsid to capsid}

For all the well-studied dsDNA phages-T4, lambda, $\mathrm{P} 22$, $\mathrm{P} 2 / \mathrm{P} 4$ and HK97-the maturation transition is an irreversible conformation change of the viral shell

*Corresponding author. Tel.: + 1-617-253-4700. Email: jaking@mit.edu

ๆ Tel.: + 1-617-253-4465. Email: gossard@ mit.edu. 

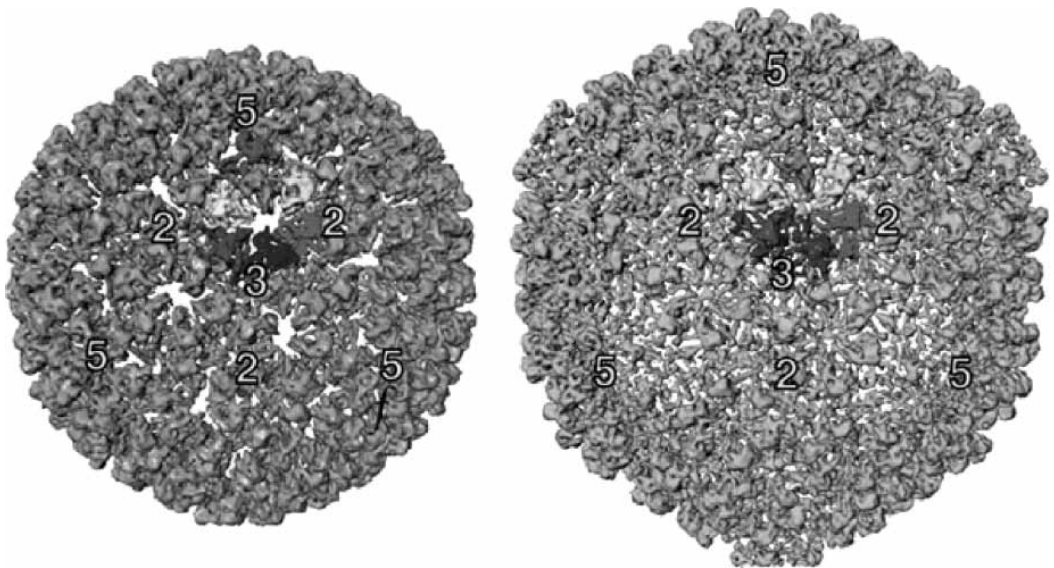

Figure 1. Cryo-EM images of P22 procapsid and capsid are on the left and right, respectively. The 5-fold, 3-fold and 2-fold axes of symmetry are highlighted. Coat proteins making up the asymmetric unit are shown in colour. From [7].

characterized by an increase in radius and a more polyhedral appearance as shown in figure 1. In fact, studies of the transition by calorimetry reveal that the procapsid is in a metastable higher energy state, and the transition to the mature lattice is exothermic [5]. Though the procapsid-to-capsid transitions have been well described, structural data has been limited to the medium resolution obtainable from cryo-electron microscopy. However, the structure of one dsDNA phage, Hong Kong 97 of $E$. coli has been crystallized and the capsid subunits structure determined to high resolution by [14]. The HK97 viral capsid comprises 420 sub-units in a $T=7$ packing arrangement. The structure of the HK97 capsid is unusual (perhaps unique) in that its coat protein chains are intertwined and cross-linked in a so-called "chain-mail" topology as shown in figure 3 .

\section{Analysis and data}

One major question concerns whether the lattice transformation is associated with a change in subunit conformation or a change in the contacts between subunits. Coat protein subunits of P22 and HK97 provide conflicting answers to this question. The coat proteins of P22 and HK97 have less than 20\% sequence identity, yet
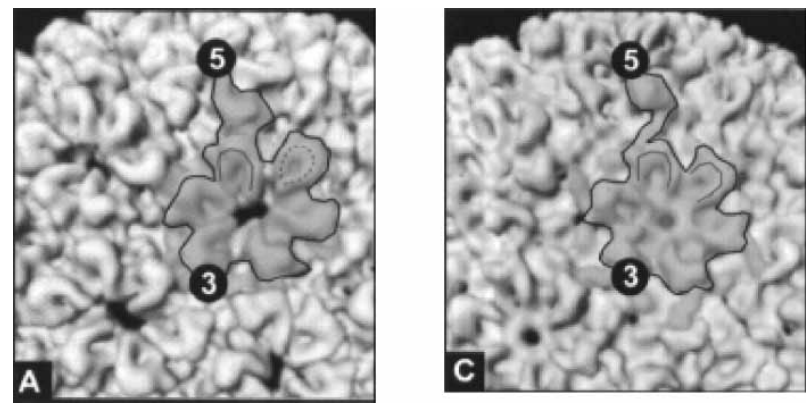

Figure 2. Cryo-EM images of P22 procapsid and capsid showing skewed hexamers becoming more regular during the maturation transition. From [15]. they both contain three major helices that are aligned to a surprising degree. Figure 4 shows the 3D structure of a HK97 subunit superimposed on a cryo-EM image of a P22 subunit. Jiang et al. [7] have shown that the major helices of P22 undergo significant movement during the maturation transition as shown in figure 5. Whether the major helices of HK97 undergo a similar motion has not yet been unambiguously resolved [3].

Another important question concerns the extent to which the coat proteins of viruses with more than 60 subunits must undergo a conformation change simply to form a closed shell with icosahedral symmetry as quasiequivalence theory suggests. To obtain some quantitative insight into this question the subunits of the HK97 capsid were examined numerically. The asymmetric unit of HK97 consists of 7 coat proteins labeled A-G, as is shown in figure 6. The 5-fold axis of symmetry is located at the apex of subunit $\mathrm{G}$, at the top of the figure.

A ribbon diagram of one of the subunits is shown in figure 7. The subunit consists of a central domain containing three alpha helices, the largest of which is designated "H1" here. Protruding from the central domain are two extended arms. The first, designated "a", is the Nterminus and the second, designated " $b$ " is an extended loop. Two additional regions, a loop near the apex, and a nearby alpha helix, are designated "c" and " $d$ ", respectively.

We assumed that the major helix, H1, was relatively rigid with respect to the remainder of the protein sub-unit. Consequently, three alpha-carbons on H1 (residues 204, 222 and 210, respectively) were used to define a local coordinate system on each subunit. The coordinate system and the backbone of one of the subunits are shown in figure 8 .

Rigid-body transformations were applied to all seven subunits so as to make the coordinate systems coincide and therefore, superimpose the subunits to admit a visual comparison of their conformations. Figure 9 shows this superposition of the backbones of all seven subunits. This figure gives a qualitative sense of the extent to which the 


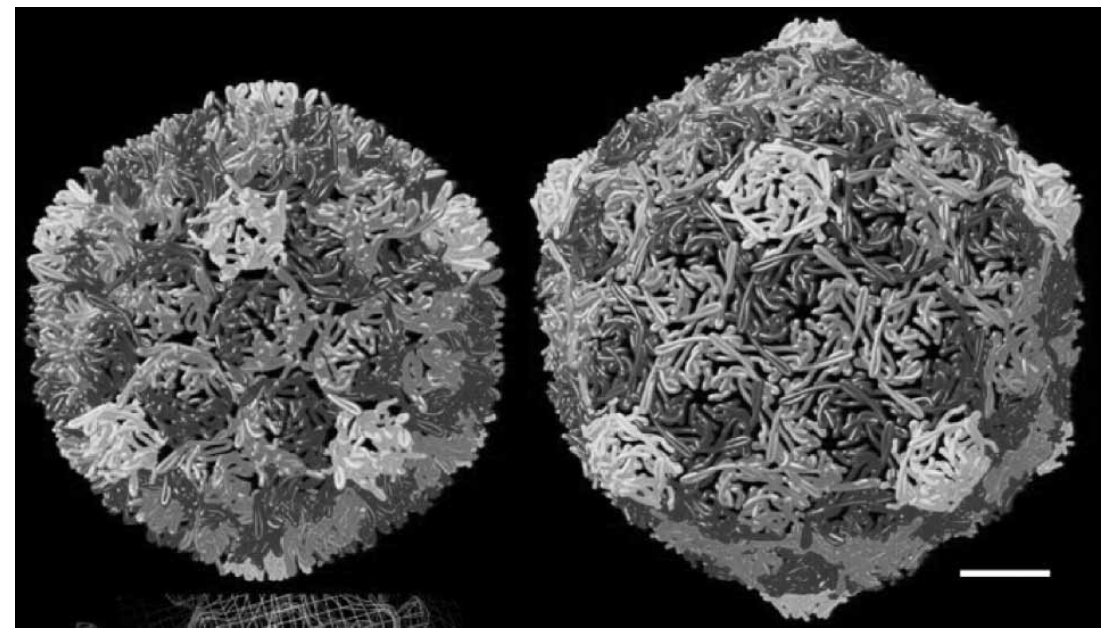

Figure 3. HK97 maturation transition. The image on the left is the procapsid. The image on the right is the capsid. Note the larger radius and more angular shape of the capsid. From [3].

subunits' conformations diverge from the single original (pre-assembly) conformation.

To obtain a more quantitative understanding, the coordinates of the alpha-carbons for the six subunits forming the hexamers in the asymmetric unit were averaged and used as a reference. Then for each subunit, the "error" or "deviation" was defined as the Euclidean distance between each alpha-carbon from the reference, i.e. the hexameric average. This "deviation" was plotted as a function of residue number, i.e. backbone length, for each subunit as shown in figure 10 .

It should be noted that the "deviations" found in this way are all positive because only the distance between each alpha-carbon and the reference was determined, i.e. there is no sign information.

From figure 10, it can be seen that the maximum deviation occurs at four points along the backbone of each subunit. These are designated "a", "b", "c" and "d", respectively and can also be seen in figure 7 . From figure 10 , it can also be seen that the largest deviations occur at positions "a" and "b" and on subunits G, A and B, respectively. This is to be expected given the higher curvature of the capsid shell near its 5-fold axes of symmetry.
To better understand the collective motion of the HK97 subunits, a 3-D "disk" was superimposed on the major helices of each subunit. The result is shown in figure 11. The images on the upper left and upper right show the relative orientation of the subunits of the HK97 procapsid. The images on the lower left and lower right show the relative orientation of the subunits of the HK97 capsid. The skewed nature of the hexameric grouping in the procapsid is readily apparent as is the more regular nature of the hexameric group in the capsid. In the capsid, all seven subunits of the asymmetric unit are extended and lie essentially in the same plane. Consequently, the asymmetric unit covers a larger area that contributes both to the larger radius and larger volume of the capsid shell. Further, the planar shape of the asymmetric unit results in the major curvature of the capsid shell lying at the interfaces between asymmetric units. In the procapsid, subunit $\mathrm{G}$ is tilted with respect to the hexameric group and the subunits in the hexamer are rotated into a more compact, less extended configuration. The asymmetric group as a whole is smaller and more compact and the curvature of the procapsid shell is distributed over the asymmetric unit as well as at the interfaces between asymmetric units.
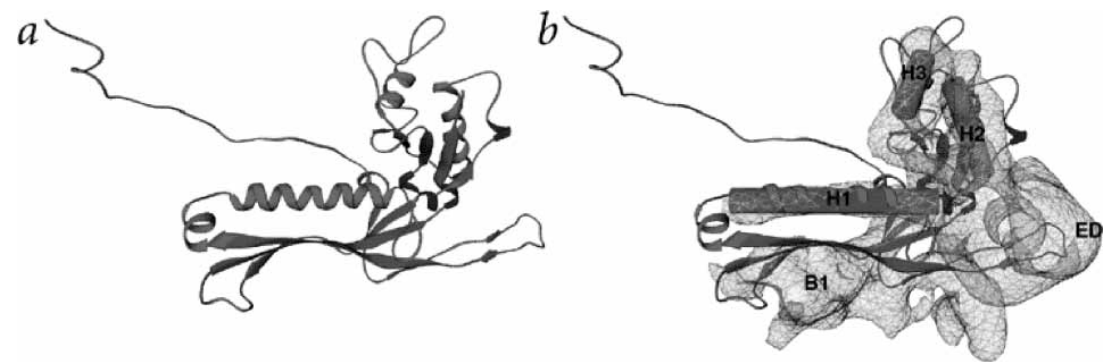

Figure 4. Structure of HK97 subunit superimposed on cryo-EM image of P22 subunit. HK97 and P22 subunits both have three major helices and a surprising degree of alignment despite having less than $20 \%$ sequence identity. From [7]. 


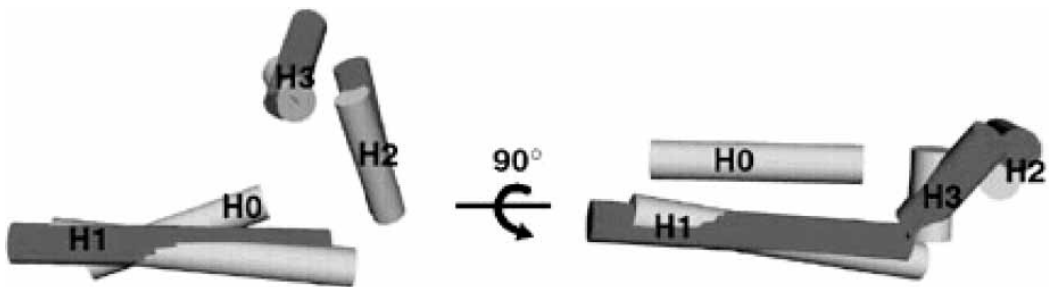

Figure 5. Movement of P22 subunit helices during maturation transition. Light cylinders represent the location of P22 subunit helices in procapsid. Dark cylinders represent the location of P22 subunit helices in the capsid. The image on the right is rotated $90^{\circ}$ from the image on the left. From [7].

\section{Conclusions and recommendations}

The changes in subunit conformation associated with the procapsid-to-capsid transformation are significant, but not outside the range of protein motions observed for polymerases, ATPases, and other protein complexes. However, the changes discussed above are not occurring free in solution, but within the capsid lattice. The collective motion of a set of subunits involves both rotations and translations as can be seen in figure 11. We do not yet understand the coupling between the individual motions of the subunits and the collective motions within the lattice.

\section{Functions of the lattice transitions}

Phages P22 and HK97 are not closely related, and the amino acid sequences of their coat subunit polypeptide chains are not obviously homologous though they have the same basic fold. Yet they participate in a very similar rearrangement reaction in the procapsid-to-capsid transition. This suggests a biologically conserved function. We suspect that the functions of this transformation have to do with the properties and functions of the inner surface of the capsid lattice. In the procapsid, sites on the inner surface are occupied by scaffolding proteins. When the scaffolding subunits exit, some parts of these sites

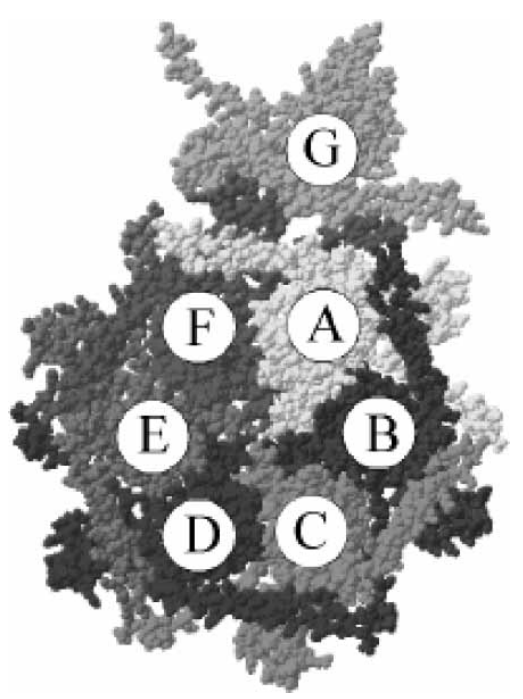

Figure 6. The asymmetric unit of the HK97 viral capsid (PDB ID: 1fh6). The "chain-mail topology" refers to the interlocking nature of the subunits. The 5-fold axis of symmetry is located at the apex of subunit G. may be available for contact with the entering DNA. In the mature capsids of P22, Lambda, T4 and other phages, there is no evidence that the packaged DNA is actually interacting with the inner lattice of the capsid. However, that might not be true when the first turns of DNA are pumped into the procapsid, particularly if this takes place before the procapsidto-capsid lattice transition. Because of the stiffness of DNA, the initial stretches of DNA injected would be expected to take the maximal curvature allowed by the shell diameter ( $~ 60 \mathrm{~nm}$ ), and thus pack against the inside of the capsid [4].

The packed DNA appears to be organized as concentric shells [15]. Specific binding interactions might be important in organizing the initial coils of DNA. However, such binding interactions, though helping to organize the entering DNA, would interfere with the subsequent release of packaged DNA and its injection into the host cell. In this context, the role of the lattice transition is to terminate transient interactions with the outer coils of DNA, releasing the DNA within the inner volume of the capsid, ready for release into the cell during DNA injection.

\section{Initiation and propagation of the lattice transition}

DNA enters the precursor shell through the portal complex. It seems reasonable that events happening at this complex initiate

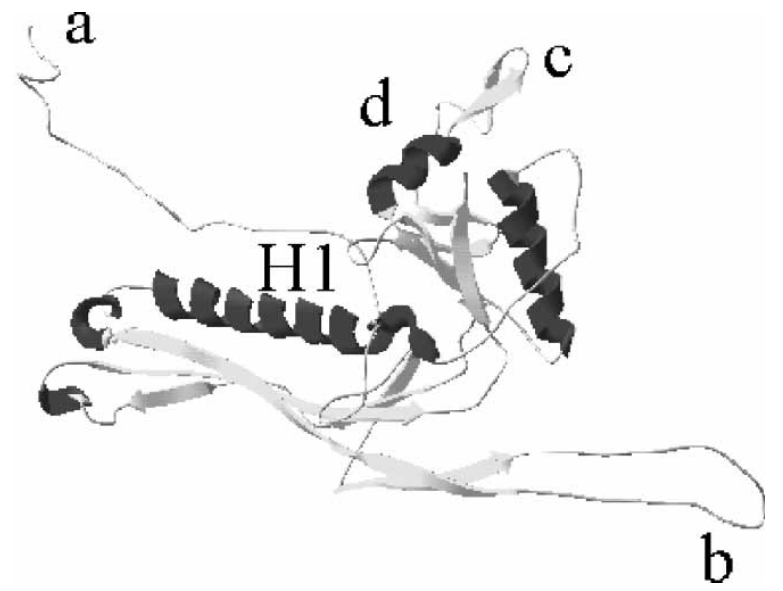

Figure 7. A ribbon diagram on a single coat protein of the HK97 viral capsid (1fh6). The coat protein subunit consists of a central domain containing three alpha-helices, the largest of which is designated "H1" here. Protruding from the central domain are two extended arms. The first, designated "a", is near the N-terminus and the second, designated "b", is an extended loop. Two additional regions, a loop near the apex, and a nearby alpha-helix, are designated "c" and "d", respectively. 


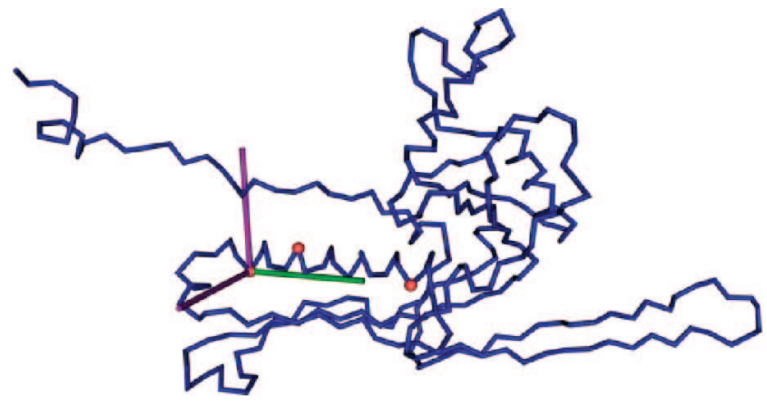

Figure 8. The backbone of a single coat protein of HK97 viral capsid. Three alpha-carbons (residues 204, 222 and 210, respectively, designated by small spheres) on the largest alpha-helix were used to define a local coordinate system.

the procapsid to capsid transition. A considerable quantity of ATP is hydrolyzed in this process and substantial mechanical forces are generated. We envisage that the initiation of pumping of the DNA into the procapsid is the stimulus that starts the transition, which propagates from the portal vertex.

Analysis of the beginning and end states does not reveal the pathway of the propagation of the lattice transition. It could proceed helically around the lattice, one hexamer at a time. Or alternately, it could proceed with five-fold symmetry, up all five icosahedral faces, terminating at the vertex opposite to the portal vertex. Sorting this out will require trapping intermediates in the transition, which is feasible by cryo-EM.

Many steps in viral capsid assembly appear to proceed independently of the cellular apparatus. These steps are potential targets for antiviral drugs. Indeed the actual inhibitory effectiveness of the anti-HIV protease follows from the role of this protease in generating the nucleocapsid

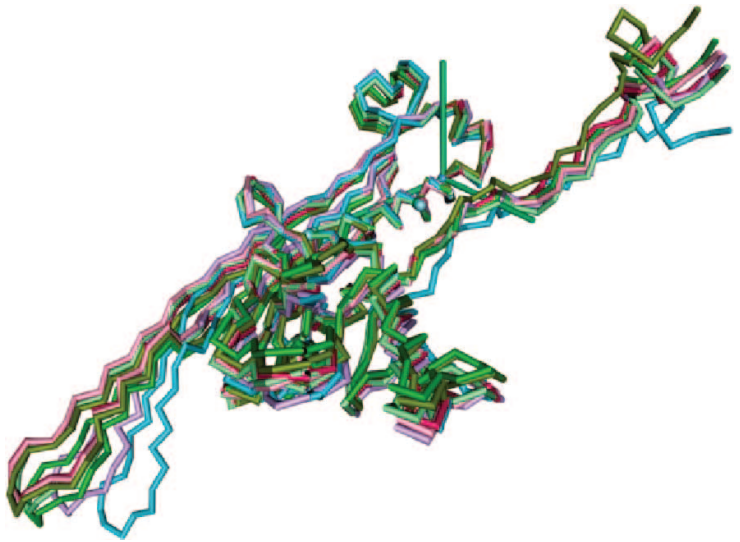

Figure 9. Rigid-body transformations were applied so as to superimpose the subunits to admit a visual comparison of their conformations. This figure gives a qualitative sense of the extent to which the subunits' conformations diverge from the putative single original (pre-assembly) conformation.

proteins. For viruses such as Herpes virus, the scaffolding is removed by proteolytic functions, and is a possible target for anti-virals. However other steps, such as DNA packaging, or the essential capsid transitions discussed here, open up other targets.

\section{Acknowledgements}

We thank Wah Chiu, Jack Johnson and Roger Hendrix for valuable discussions and access to data, Chris Mitchell of MIT for assistance in the modelling and Peter Weigele for assistance with the manuscript.

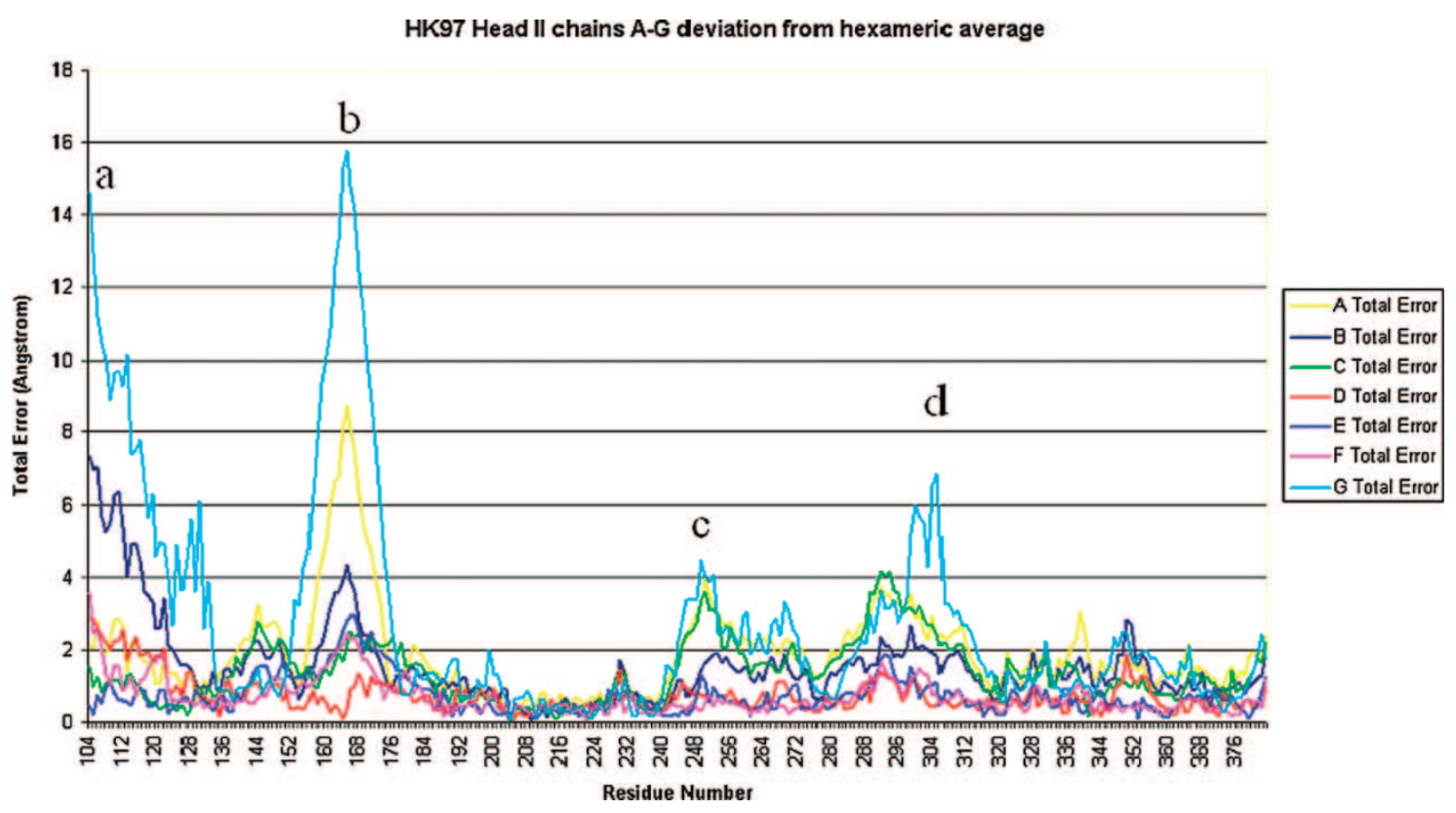

Figure 10. The coordinates of the alpha-carbons for the 6 subunits forming the hexamer in the asymmetric unit were averaged (subunits A-F in figure 6). Then for each subunit, the "error" or "deviation" was defined as the Euclidean distance between each alpha-carbon from the reference, i.e. the hexameric average. This "deviation" is plotted as a function of residue number, i.e. backbone length, for each subunit. The largest deviation occurs at 4 points along the backbone of each subunit. These are designated "a", "b", "c" and "d", respectively. Their physical location on the subunit can be seen in figure 2. The largest deviations occur on subunits G, A and B, respectively, and at positions "a" and "b". 

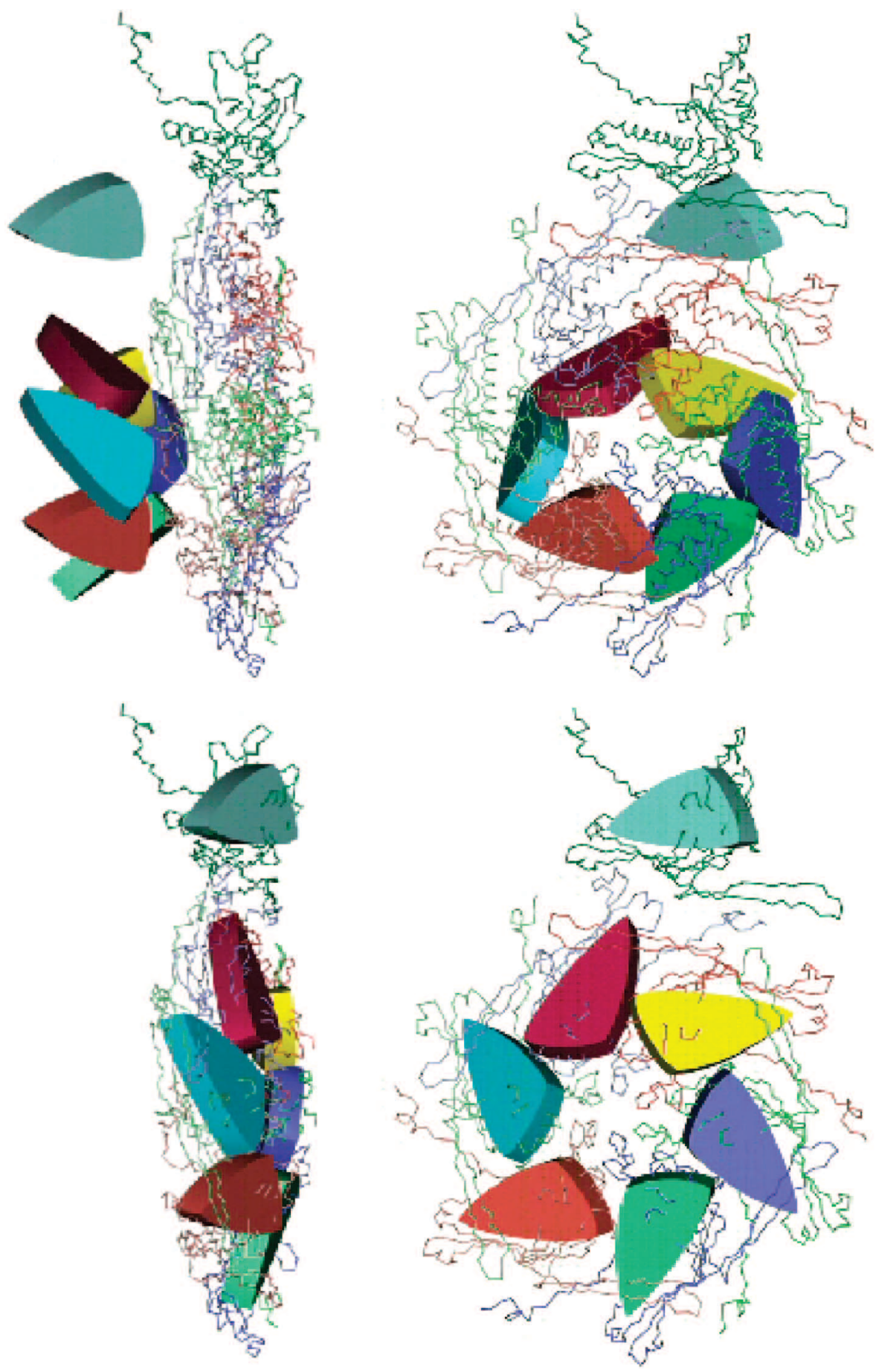

Figure 11. Maturation transition of HK97 (1fh6). To facilitate visualization, 3D discs have been superimposed on the three major helices of the subunits. Upper left: side view of procapsid. Upper right: front view of procapsid. Lower left: side view of the capsid. Lower right: front view of the capsid.

\section{References}

[1] Baker, T.S., Olsen, N.H. and Fuller, S.D., 1999, Adding the third dimension to virus life cycles: three-dimensional reconstruction of icosahedral viruses from cryo-electron micrographs. Microbiol. Mol. Biol. Rev., 63, 862-922.

[2] Caspar, D.L.D. and Klug, A., 1962, Physical principles in the construction of regular viruses. Cold Spring Harb. Symp. Quant. Biol., 27, 1-24.

[3] Conway, J.F., Wikoff, W.R., Cheng, N., Duda, R.L., Hendrix, R.W., Johnson, J.E. and Steven, A.C., 2001, Virus maturation involving large subunit rotations and local refolding. Science, 292, 744-748.
[4] Earnshaw, W.C., King, J., Harrison, S.C. and Eiserling, F.A., 1978, The structural organization of DNA packaged within the heads of T4 wild-type, isometric and giant bacteriophages. Cell, 14, 559-568.

[5] Galisteo, M.L. and King, J., 1993, Conformational transformations in the protein lattice of phage P22 procapsids. Biophys. J., 65, 227-235.

[6] Horn, T., Wurtz, M. and Horn, B., 1976, Capsid transformation during packaging of bacteriophage (DNA). Philos. Trans. R. Soc. Lond. B. Biol. Sci., 276, 51-61.

[7] Jiang, W., Li, Z., Zhang, Z., Baker, M.L., Prevelige, P.E., Jr. and Chiu, W., 2003, Coat protein fold and maturation transition of 
bacteriophage P22 seen at subnanometer resolutions. Nat. Struct Biol., 10, 131-135.

[8] King, J., Botstein, D., Casjens, S., Earnshaw, W., Harrison, S. and Lenk, E. 1976, Structure and assembly of the capsid of bacteriophage P22. Philos. Trans. R. Soc. Lond. B. Biol. Sci., 276, $37-49$.

[9] King, J. and Chiu, W., 1997, The procapsid to capsid transition in double-stranded DNA bacteriophages. In: W. Chiu, R.M. Burnett and R. Garcea (Eds) Structural Biology of Viruses (Oxford University Press), pp. 288-311.

[10] Lata, R., Conway, J.F., Cheng, N., Duda, R.L., Hendrix, R.W., Wikoff, R.W., Johnson, J.E., Tsuruta, H. and Steven, A.C., 2000, Maturation dynamics of a viral capsid: Visualization of transitional intermediate states. Cell, 100, 253-263.

[11] Prevelige, P.E., Thomas, D. and King, J., 1993, Nucleation and growth phases in the polymerization of coat and scaffolding subunits into icosahedral procapsid shells. Biophys. J., 64, 824-835.

[12] Sun, Y., Parker, M., Weigele, P., Casjens, S., Prevelige, P.E. and Krishna, N.R., 2000, Structure of the coat protein-binding domain of the scaffolding protein from a double-stranded DNA virus. J. Mol. Biol., 297, 1195-1202.

[13] Wagner, J. and Laemmli, U.K., 1976, Studies on the maturation of the head of bacteriophage T4. Philos. Trans. R. Soc. Lond. B. Biol. Sci., 276, 15-26.

[14] Wikoff, W.R., Liljas, L., Duda, R.L., Tsuruta, H., Hendrix, R.W. and Johnson, J.E., 2000, Topologically linked protein rings in the bacteriophage HK97 capsid. Science, 289, 2129-2133.

[15] Zhang, Z., Greene, B., Thuman-Commike, P., Jakana, J., Prevelige, P., King, J. and Chiu, W., 2000, Visualization of the maturation transition in bacteriophage $\mathrm{P} 22$ electron cryomicroscopy. J. Mol Biol., 297, 615-626. 


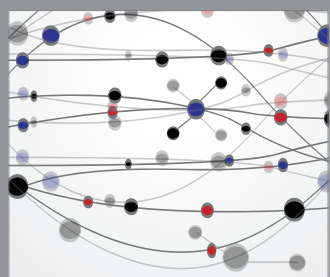

The Scientific World Journal
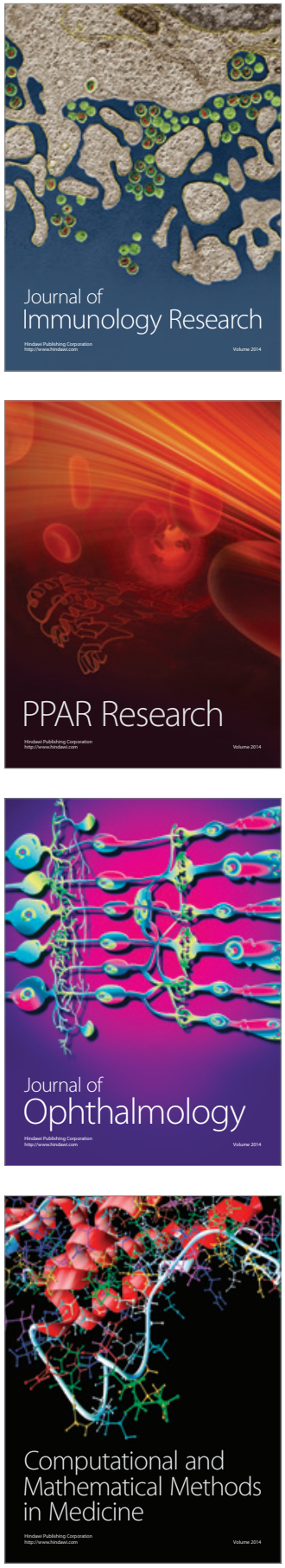

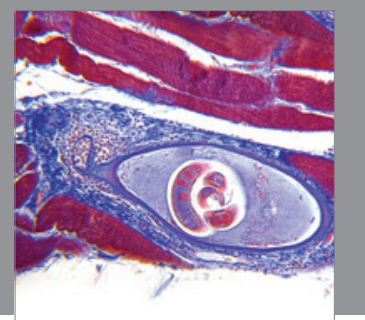

Gastroenterology

Research and Practice
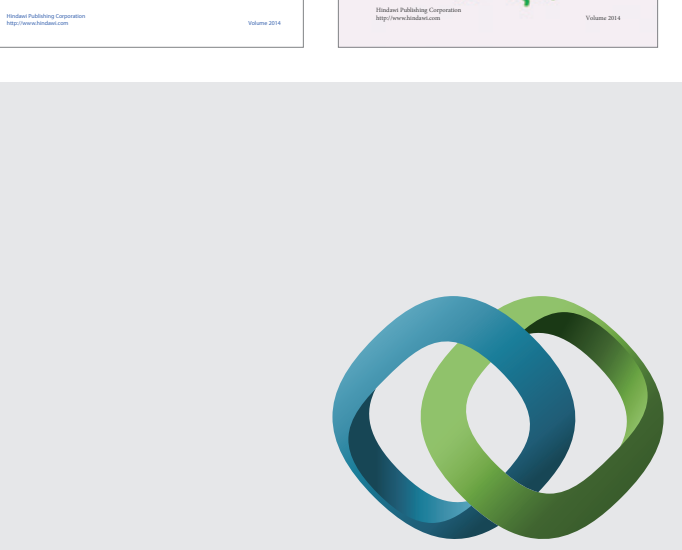

\section{Hindawi}

Submit your manuscripts at

http://www.hindawi.com
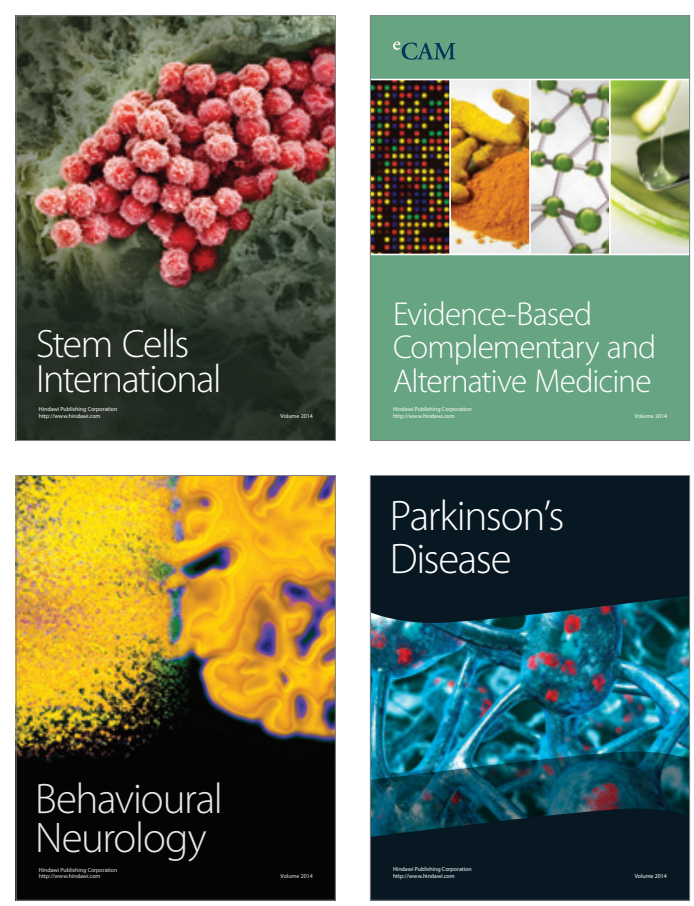

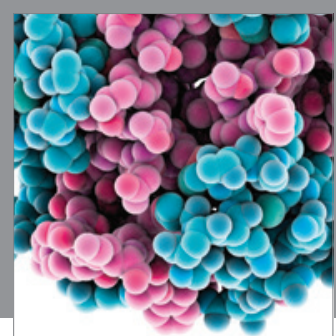

Journal of
Diabetes Research

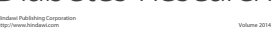

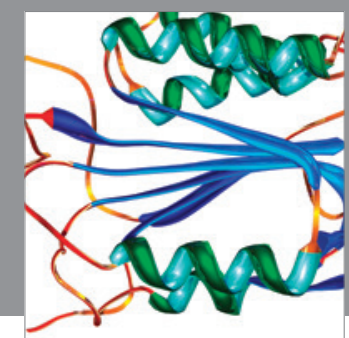

Disease Markers
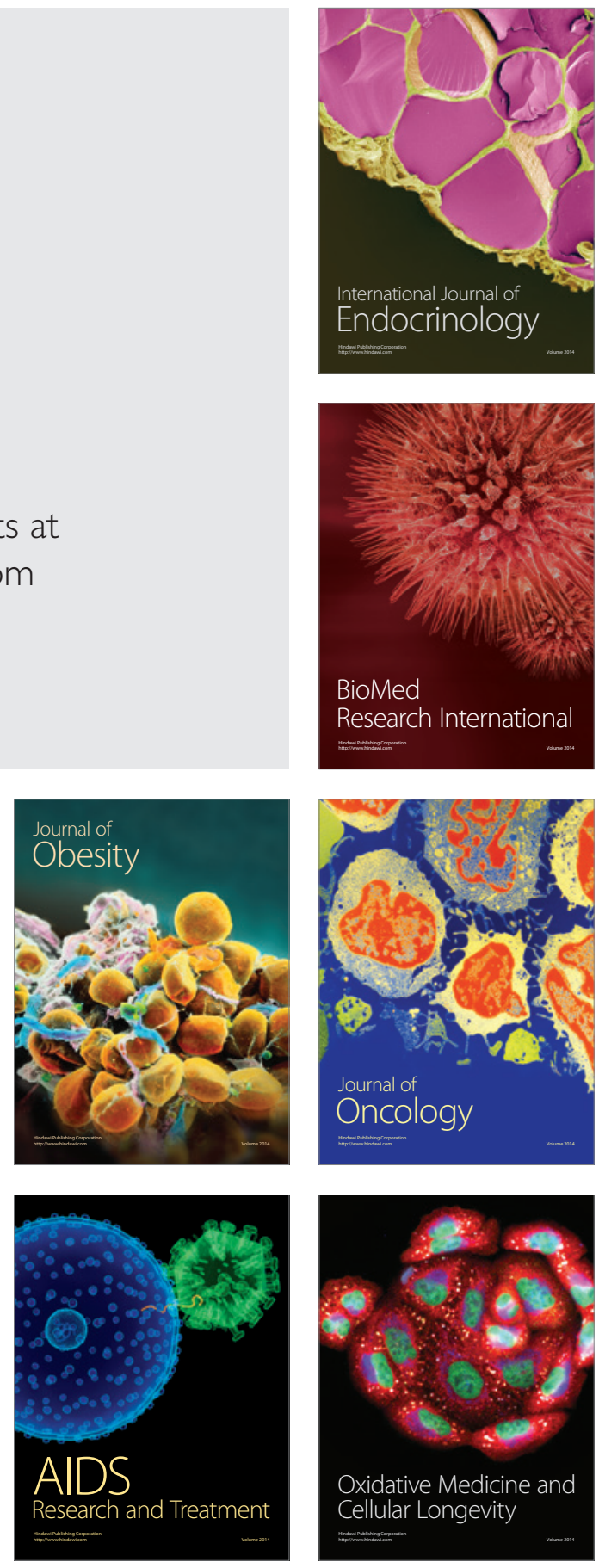